\author{
WACŁAW PAGÓRSKI \\ Uniwersytet im. Adama Mickiewicza w Poznaniu \\ E-Mail: waclaw.pagorski@amu.edu.pl \\ https://orcid.org/0000-0001-8448-6674
}

\title{
TAGUNGSBAND \\ REFORMACJA PROTESTANCKA 1517 ROKU \\ ZUM 500. JUBILÄUM DER REFORMATION
}

Der Sammelband Reformacja protestancka 1517 roku. Spory o dziedzictwo ist der erste von zwei geplanten Tagungsbänden, in denen Aufsätze einer internationalen Tagung Reformation - zwischen Gewinn und Verlust. Retrospektive - Revision - Redefinition ${ }^{1}$ gesammelt werden. Die Konferenz hatte zum Ziel, im Rahmen des 500. Jubiläums der Reformation nach ihrem bleibenden Erbe aus der Sicht der Gegenwart zu fragen und zu diskutieren. Der Sammelband umfasst 18 polnische Texte aus unterschiedlichen Disziplinen, wie Geschichts-, Kultur- Literatur- und Sprachwissenschaft sowie Theologie. Das Vorwort von Cezary Lipiński und Wolfgang Brylla ermöglicht einen guten Einstieg in das breite Spektrum der Darstellungen: Thematisiert werden nicht nur die Betrachtung der Reformation in der Geschichte sowie die wichtigsten Tendenzen der gegenwärtigen Reformationsforschung, sondern auch Ablauf und Probleme um das Begehen und Andenken des 500. Jubiläums in Deutschland und Polen. Auf Grund dieser Zusammenstellung kamen die Autoren zur Schlussfolgerung, dass die Reformation und die Person Martin Luthers immer noch extrem unterschiedlich bewertet werden sowie, dass die Bereitschaft aller Beteiligten, sich belastbares Wissen über den zeitlichen Kontext, die Ursachen, Protagonisten und Leistungen der Reformation anzueignen, sehr gering ist. Deswegen gilt es als Ziel für

${ }^{1}$ Reformacja protestancka 1517 roku. Spory o dziedziectwo, hg. v. C. Lipiński, W. Brylla, (2018), 342 S., ISBN 978-83-65815-16-3. Die Tagung fand im Oktober 2017 in Grünberg (Zielona Góra) statt und wurde von den Instituten für Germanistik der Universität Zielona Góra und der Adam-Mickiewicz-Universität Poznań (Posen) organisiert. 
diesen Band, auf das vielfältige Erbe der Bewegung mit Beispielen aus der Vergangenheit und Gegenwart hinzuweisen. Der Hauptteil wird in fünf Sektionen aufgeteilt: Querschnittsauffassungen, Im Kreis der religiösen und theologischen Auswirkungen, In der Sphäre der historischen Fragen, Im Paradigma der christlichen Kultur und Im Bereich der Sprache.

Die Texte der Sektion Querschnittsauffassungen dienen als eine Art Rahmen für die anderen Beiträge, in dem ihre Autoren die Reformation in der globalgeschichtlichen Perspektive betrachten. Im Aufsatz von Heinz Schilling unter dem Titel 1517: Mönch und Rhinozeros. Weltgeschichte eines Jahres wird die Reformation mit anderen Ereignissen aus der Weltgeschichte verortet, wie zum Beispiel den vorlutherischen Klerusreformen in Spanien, dem Einzug des künftigen Kaisers Karl V. in Valladolid oder der Eroberung des Reichs der Mameluken durch die Osmanen. Eine solche Kontextualisierung befreit, so die Stellungnahme des Autors, die Reformation vom falschen Mythos, dass mit Luther die Neuzeit beginne. Eine ähnliche Perspektivierung bietet Wolfgang Reinhard in seiner Abhandlung Reformation global?, indem er die Reformation mit anderen religiösen (Erneuerungs-)Bewegungen in Indien, China sowie im Nahen Osten und Judentum dieser Zeit in Verbindung setzt, die unabhängig voneinander verliefen. Es werden aber auch mehrere Beispiele für die globale Rezeption und Anspielungen auf die Reformation im Zeitalter des Kolonialismus geschildert.

Die zweite Sektion, Im Kreis der religiösen und theologischen Auswirkungen, setzt sich aus fünf Beiträgen zusammen. Der erste Text von Jacek Froniewski mit dem Titel Einfluss des ökumenischen Dialogs auf die Reinterpretation Luthers in der Katholischen Kirche handelt vom Wandel des katholischen Lutherbildes in der offiziellen Kirchenlehre. Nach der kurzen Skizze der Periode vor dem Vaticanum II werden Auszüge sowohl aus den Konstitutionen des Konzils sowie Dokumente der katholisch-evangelischen Kommission als auch Aussagen der drei letzten Päpste vorgeführt. Auf Grund ihres eingehenden Studiums stellt der Autor fest, dass die Entwicklung des offiziellen Lutherbildes seit dem II. Vatikanischem Konzil ein beeindruckender Prozess sei, wobei das volkstümliche Lutherbild in Polen mit der offiziellen Lehre der Katholischen Kirche nicht immer übereinstimmt.

Die nächste Abhandlung ist der Situation der Lutheraner in Schlesien im 17. Jahrhundert gewidmet. Cezary Lipiński beschreibt in seinem Aufsatz 
Die Krise der lutherischen Spiritualität im Schlesien des 17. Jahrhunderts zuerst die konfessionelle Situation in Schlesien im genannten Zeitalter. Auf dieser Basis analysiert er drei Beispiele für damalige ablehnende Stimmen gegen die lutherische Orthodoxie (Johann Christian von Brieg, Abraham von Franckenberg, Johann Scheffler). Lipiński will auf Grund der angeführten Diskussionen auf die innere Schwäche des damaligen Luthertums hinweisen, die den Grund für die Rekatholisierung Schlesiens vorbereitet hätte.

Noch weiter in die Geschichte greift Janusz Podzielny in seinem Beitrag zum Thema Martin Luther und die Wirtschaft. Der Autor stelle am Anfang fest, dass es „Luther nicht gelungen ist, ein kohärentes System der Wirtschaftsethik zu schaffen“; Luther knüpfte aber im Rahmen der Seelsorge mehrmals an Themen wie Handel, Gewinn, Wucher, Arbeit oder Berufung an. Im Aufsatz wird Luthers Auffassung dieser Begriffe auf Grund seiner Schriften, Predigten und Tischreden analysiert.

Der nächste Beitrag befasst sich mit Albert Schweitzers Interpretation vom Corpus Paulinum. Dorota Brylla zeigt auf Grund der Analyse „Der Mystik des Apostel Paulus" die Unterschiede in der Deutung der Paulus Briefe bei Schweitzer und Luther. Die mystische Erfahrung spiele für den deutsch-französischen Theologen und Arzt eine zentrale Rolle im christlichen Leben, und die klassische Interpretation der lutherischen Rechtfertigungslehre (sola gratia per fidem) werde durch die Idee des mystischen „In-Christus-Sein“ ersetzt.

Am Ende der ersten Sektion wird ein Beispiel für die Rezeption Luthers in der Orthodoxen Kirche geschildert. Andrey Kotin stellt in seinem Beitrag mit dem Titel Reformation und Orthodoxie. Martin Luther nach der Auffassung von Dmitri Mereschkowski das Lutherbild eines russischen Schriftstellers aus der Zeit des Zweiten Weltkrieges dar. Zwei Aspekte sind bei der Analyse von Bedeutung: der mystische Historismus bei Mereschkowski und seine Fokussierung auf die Kategorie der Person. Kotin hebt hervor, dass das analysierte Buch des russischen Autors, mit dem Titel Reformatoren: Luther, Calvin und Pascal nicht als eine Biographie konzipiert war; Mereschkowski suchte bei Luther Bezugspunkte zur damaligen Gegenwart. Der Wittenberger galt ihm als „Herold der Endzeiten“, dessen Rolle darin beruhe, Orthodoxie, Katholizismus und Protestantismus wiederzuvereinigen.

In der Sphäre der historischen Fragen, so heißt die dritte Sektion des Sammelbandes, die aus drei Beiträgen besteht. Zwei von ihnen handeln von der 
Reformation in der Lausitz, wobei die Akzentlegung jeweils unterschiedlich ausfällt. Im Aufsatz von Tomasz Jaworski (Reformation in der Lausitz - ihr Verlauf und ihre Bedeutung) wird der Frage nach der Vielfältigkeit der Ursachen und den Auswirkungen der Reformation sowie den unterschiedlichen Konfessionalisierungen in der Lausitz nachgegangen. Der Autor beachtet dabei den national-ethnischen Aspekt besonders stark und weist darauf hin, dass es in manchen Teilen der Region im Laufe der Konfrontation mit dem Luthertum zum Erwachen der ethnischen Identität und zur Stärkung des Katholizismus kam. Piotr Haracz wendet in seinem Beitrag eine engere Perspektive an und befasst sich nur mit dem östlichen Teil der Niederlausitz. In einer Mikroanalyse zeigt er den Verlauf der Reformation am Beispiel einzelner Ortschaften auf und zieht daraus den Schluss, dass im Endeffekt in ca. 40 Jahren fast alle Gemeinden bzw. Ordenseinrichtungen in dieser Region lutherisch wurden.

Diesen Teil beschließt der Beitrag von Katarzyna Wójcik über die Geschichte der deutschen Evangelischen aus dem Cholmer und Lubliner Lande in Kurt Lücks Heimatbuch „Die deutschen Siedlungen im Cholmer und Lubliner Lande“. Den Text eröffnet eine kurze Skizze über die Entwicklung der deutschen lutherischen Gemeinden in der genannten Region, beginnend mit der ältesten Migration in der vorletzten Dekade des 18. Jahrhunderts. Im weiteren Teil befasst sich die Autorin mit der Person Kurt Lücks - eines deutschen Historikers, Minderheitsvertreter und späteren SS-Hauptsturmführers - und seinem Heimatbuch über die deutschen Siedlungen zwischen den Flüssen Wieprz und Bug sowie dem Gebrauch des Buches durch die NS-Propaganda.

Die nächste Sektion Im Paradigma der christlichen Kultur beinhaltet sechs Beiträge. Der erste von ihnen, Der Mensch des Jahrhunderts? Die Bedeutung Martin Luthers für die Vormärz-Bewegung aus der Perspektive von Hoffmann von Fallersleben von Mariusz Dziewczyński handelt von der Rezeption Luthers bei August Heinrich Hoffmann von Fallersleben. Auf Grund der Analyse seiner Texte und Vorträge wird gezeigt, dass der Germanist und Professor der Breslauer Universität zwar die Bedeutung des Reformators besonders im Bereich der Entwicklung der deutschen Sprache pries, jedoch den apolitischen Charakter von Luthers Schaffens kritisierte.

Bezogen auf das 19. und frühere 20. Jahrhundert ist der nächste Text mit dem Titel Protestantismus in der Auffassung von Carl Mund und den 
Autoren der Zeitschrift „Hochland“. Marek Jakubów zeigt ausgewählte katholische Stellungnahmen aus der Zeit der Jahrhundertwende, welche die literarischen Texte protestantischer Autoren wie auch die Person des Reformators selbst in einer teilweise überkonfessionellen Perspektive erfassten.

Auf das Erbe der Reformation in Polen und die Möglichkeiten seiner Erforschung weist Jakub Z. Lichański hin. Der Aufsatz mit dem Titel Die gesellschaftlichen und intellektuellen Eliten und ihr Einfluss auf die Entwicklung des Schrifttums am Beispiel der protestantischen Gesangbücher. Eine Wissensdatenbank - von den Anfängen bis 1918 beinhaltet die Beschreibung und mögliche Effekte eines geplanten wissenschaftlichen Projekts.

Die Wirkung der Reformation wird auch im Kontext der Entwicklung des allgemeinen Zugangs zur Bildung thematisiert. Rafał Werszler präsentiert Beispiele in seiner Abhandlung zum Einfluss der Reformation auf die das Lesen popularisierende Transformationsrichtung der Gestaltung von evangelischen Librarien und Repositorien des 16. und 17. Jahrhunderts in Schlesien, welche Änderungen im Bereich der Gestaltung der Innenräume, Ausstattung sowie der Bereitstellung von Büchern unter dem Einfluss der Reformation eingeführt wurden.

Der nächste Beitrag gehört in den Bereich der imagologischen Studien. Grzegorz P. Słowik befasst sich in seinem Text Das Bild des Schweden-Protestanten in Polen der Wasa-Zeit im Lichte der ausgewählten Memoiren aus dem 17. Jahrhundert mit den unterschiedlichen Elementen - positiven wie negativen - des Schwedenbildes und betont den Zusammenhang zwischen ethischen und konfessionellen Aspekten.

Die Sektion beschließt der Beitrag von Wolfgang Brylla (Luter.pl. Andenken des 500. Jubiläums der Reformation im Spiegel von Politik, Medien und Wissenschaft). Es ist eine Zusammenfassung der Ereignisse und Diskussionen in Polen zum 500. Jubiläum der Reformation. Der Autor betont zwar den Unterschied im Ausmaß der Feierlichkeiten in Deutschland und in Polen, doch stellt er fest, dass die Reformation im polnischen Mainstream doch sichtbar war. Zum Beitrag wurde auch eine tabellarische Auflistung der Tagungen in Polen hinzugefügt, die dem Thema Reformation gewidmet waren.

In der letzten Sektion unter dem Titel Im Bereich der Sprache gibt es zwei Beiträge. Der erste von ihnen schildert ein frühneuzeitliches Beispiel für den Einfluss der Konfession auf die Sprache. Magdalena Hawrysz analysiert in ihrer Abhandlung Die arrianischen religiösen Begriffe im Register zum Neuen 
Testament in Übersetzung von Marcin Czechowic aus dem Jahre 1577 Begriffe aus dem Feld der Doktrin, des Kults sowie der religiösen Organisation und Erfahrung und zeigt die Strategien der Übersetzung sowie die Rolle der Weltanschauung bei der Gestaltung der religiösen Texte in der frühen Neuzeit auf. Zurück in die Gegenwart trägt den Leser Marcin Miodek, der in seiner Studie Das Schlagwort „Luter" in den polnischen Enzyklopädien und Universallexika - ausgewählte Aspekte 36 Einträge über den Reformator zusammenstellt und vergleicht. Unter die Lupe nimmt er sowohl die ersten großen Werke aus dem 19. Jahrhundert als auch neue Publikationen. Auf Grund des Vergleichs stellt der Autor einerseits mehrere Diskrepanzen zwischen den Beiträgen, aber andererseits auch Beispiele für die Wiederholung alter Mythen sogar in gegenwärtigen Enzyklopädien fest.

Die Tatsache, dass in dem Sammelband zwei Beiträge den einzelnen Jahren (1517 und 2017) gewidmet sind, ist symbolisch. Diese Klammer verbindet alle Beiträge aus fünf Sektionen und betont den gleichen Ausgangspunkt aller Texte - das Erbe der Reformation. Die Aufsätze charakterisieren selbstverständlich die große Vielfältigkeit in der Thematik, Methode sowie in Bezug auf den untersuchten Zeitraum. Auf den ersten Blick kann es sogar bei dem Leser zur Verwirrung führen. Auch die Reihenfolge der Beiträge innerhalb der Sektionen ist nicht immer leicht nachvollziehbar. Doch das Ziel der Herausgeber, das breite Spektrum der Wirkung der Reformation darzustellen, wurde erreicht und die Neugierde auf weitere Studien, u. a. auf den kommenden zweiten Band, geweckt. 